\title{
The Treatment of Leprosy.
}

\author{
D. F. BAXTER. \\ A Paper read at the Calcutta Leprosy Conference on 27th \\ March, 1933.
}

(Reprinted from " Leprosy in India," January, 1934).

$7 \mathrm{HE}$ main lines of the subject can be conveniently divided into three heads.

First of all, what are the directions along which institutions with no facilities for research and experiment should direct their treatment?

Secondly, where facilities for experiment are available, what should be the treatment?

Lastly, we shall consider briefly the results of treatment.

1. In the first place it may be stated that the treatment at the smaller treatment centres, and particularly the out-patient clinics, should be such as to combine ease of administration with economy and effectiveness.

Hydnocarpus Oil and its derivatives are the drugs that have received the most attention, and rightly so, because of all the numerous drugs that have been tried, they have given the most consistently satisfactory results, in the average case. Because of expense, the pure oil may have to be relied on entirely. The Medical Stores in Madras are preparing and selling the ethyl-esters at Re. 1 per pound. Such a price makes its use possible for the smaller outpatient clinics and dispensaries. The ester is stable in quality, easy to inject and suitable for intra-dermal administration. It may be found that a mixture formed by the addition of olive oil is better tolerated by certain highly infected skin cases. As a preservative, creosote is necessary.

2. For the institutions with facilities for experiment and research, certain centres should be chosen to try out any preparation that may have offered prospects of therapeutic advance. One difficulty is that patients with faith in the present treatment are with difficulty persuaded to co-operate in an experiment with a new drug whose efficacy is unknown. It is probable that this difficulty may be partly overcome through a frank explanation being given beforehand, and by asking for volunteers.

In such a way it may be possible to carry out, in certain centres, the crucial control-experiment of keeping two batches of similar patients under identical conditions, giving one batch general treatment only, and the other batch general treatment plus special leprosy treatment. 
The result would demonstrate the value or otherwise of special treatment. It may be possible to compare the findings so obtained in an in-patient institution, with a third group of out-patients, to represent the benefits of treatment without any general treatment at all, beyond what can be advised to certain cases.

3. Results of Treatment.-There are several considerations and possibilities that make the question of evaluating the results of leprosy treatment difficult.

(a) The course of the disease is a long one and slow one. The progress of the disease is usually so slow that it is often impossible to be certain from clinical examination whether a case is improving or is just stationary or is getting worse. Bacteriological findings do give a certain amount of assistance. The general impression gained from watching most cases of leprosy is that one is watching an exceedingly slow-moving condition and thus one may consider a case as "disease-arrested," when the disease is still active.

Such an error is obviously easy when one considers that the disease is essentially one of the lymphatic system. That being so, it is the activity or non-activity of the lesions in the lymphatic glands that determines permanent quiescence or not. And we have no methods of judging with certainty the activity or non-activity of such lesions.

(b) In addition to the course of the disease being a slow one, it is possible that there is a swinging character about the active physical signs. The lymphatic system is chronically involved, but skin and nerve tissue obtain floods of bacilli from time to time. These are repeatedly killed off or reduced to such a state that they are invisible. During such periods of latency, the case may be sent away as a "disease-arrested" case, to return some time later, with a relapse. Such an alternating condition of activity and latency may be found to be the normal course of events in the average case of leprosy, especially in the skin type. Naturally permanent quiescence is possible at any one of these clinically and bacteriologically latent periods. The only way of learning the truth of this surmise is the observation of large numbers of cases, receiving treatment over periods of ten to fifteen years, along with controls not receiving treatment at all.

(c) The third difficulty is that the observation period of six months, which we now consider necessary for the granting of a discharge certificate, is too short a period. Thus cases leave too soon after treatment, and relapses 
occur, but they are not discovered owing to the fact that they do not return. A good number of the relapse cases at Chingleput are those who have been compelled to return for varying reasons. To lengthen the observation period by doubling it, would be almost to halve the annual turn-over of cases in institutions that already find it difficult to cope with the large numbers desiring admission.

What are going to be the criteria for the efficiency of the treatment? It can be stated with confidence that it is very difficult to draw any conclusions from the results of treatment in purely nerve cases, because we have no way of discovering whether a nerve lesion is an active lesion or not before treatment is actually commenced. But in the skin case, pure or mixed with nerve symptoms and signs, we do have an accurate test, it being taken for granted that the presence of bacilli indicates activity.

Therefore, there will be concentration on the skin case in most figures that will be presented. It may be argued that such concentration is putting an unfair strain on the reputation of our treatment. Such is probably so, but my main object is to show the limitations of the treatment, particularly in the skin case. It is impossible to state with confidence whether treatment prevents the incidence or onset of skin symptoms in early cases, until we have got knowledge regarding the average frequency of such progression from nerve to skin, in untreated cases.

Present population of the Settlement.

Adults. Early and middle nerve 84 or 19 per cent. Skin, all stages $\ldots 236$, 54 ", Advanced nerve ... 117,27 ,

Children. Early and middle nerve 36 , 40 ", Skin, all stages $\quad . . \quad 45,50$ Advanced nerve 11,10 ,

Discharges with Certificates-

In 1930. 10 skin cases obtained S.F. cert. or 1.8 per ct.

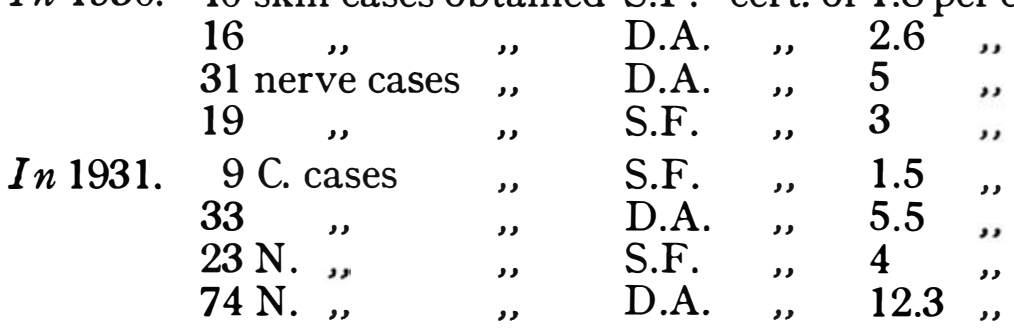

(This latter figure contains a number of burnt outs.) 
In 1932. 7 C. cases obtained S.F. cert. or 1 per ct.

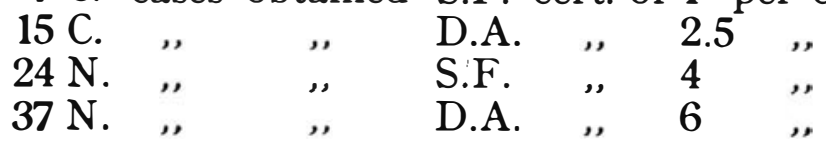

It may be noticed that in the later years a less number of skin cases have been discharged with certificates. This is due to more thorough bacteriological examinations.

Let us now examine the present-day adult population with skin leprosy and attempt to discover what has been the result of treatment on them over prolonged periods of time.

238 of the present adult inmates are skin cases, who have been in the Settlement for varying lengths of time, and have remained so ever since they were admitted.

30 cases have had treatment for 7 years and over.

10

13

15

20

34

62

54

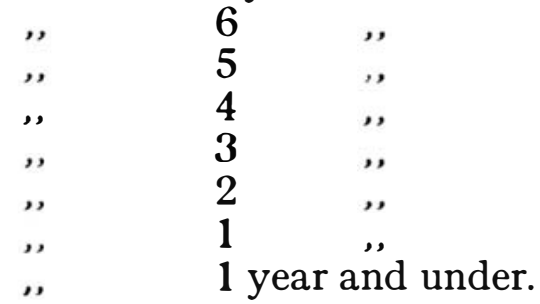

1 year and under.

Four cases not in this series were originally pure nerve cases, but have developed into the skin type, while under treatment.

74 skin cases have recently become bacilli-free, with the following lengths of treatment :-

\begin{tabular}{|c|c|c|c|c|}
\hline 10 & , & , & $6-7$ & ," \\
\hline 9 & ," & , & $5-6$ & ," \\
\hline 10 & ", & ," & $4-5$ & ", \\
\hline 8 & ," & , & $3-4$ & ", \\
\hline 9 & ., & ," & $2-3$ & ," \\
\hline 16 & ", & , & $1-2$ & , \\
\hline 8 & ," & ,", & $1 \mathrm{ye}$ & id under \\
\hline
\end{tabular}

Ten cases, now under treatment, are cases who have relapsed.

\section{General Conclusions-}

1. 24 per cent. of those who came in originally as skin cases became bacilli-negative under treatment with an average length of 3.2 years.

The remaining 76 per cent. have remained bacillipositive, under treatment with an average length of 2.7 years. 
Therefore, roughly one skin case in four may become bacilli-negative, in a seven-year period, with an average treatment period of 3.2 years.

2. Such a finding should make us chary of hoping that by treatment alone can leprosy be removed from any given district.

3. Therefore the basis of our attack on the disease must rest, until some more effective remedy is found, on voluntary isolation, either at home when possible, or elsewhere.

The soundest foundation for our campaign then, is not therapeutics, but propaganda leading to the prevention of spread of infection from the infectious skin case. 